\title{
A FAMILY OF KRALL POLYNOMIALS
}

\author{
LUC VINET*, OKSANA YERMOLAYEVA ${ }^{\dagger}$, AND ALEXEI ZHEDANOV*
}

Abstract. We consider Koornwinder's generalized Jacobi polynomials $P_{n}^{\left(j, 0 ; M_{0}, M_{1}\right)}(x)$ with weight function $w(x)=x^{j}+M_{0} \delta(x)+M_{1} \delta(1-x)$ on the interval [0,1] for $M_{0}, M_{1}$ arbitrary masses and $j=0,1,2, \ldots$ We show that the polynomials $P_{n}^{\left(j, 0 ; M_{0}, M_{1}\right)}(x)$ are eigenfunctions of a differential operator of order $2 j+6$ with polynomial coefficients.

1. Introduction. Let $P_{n}(x)$ be orthogonal polynomials (OP) satisfying the three-term recurrence relation $[1]$

$$
P_{n+1}(x)+b_{n} P_{n}(x)+u_{n} P_{n-1}(x)=x P_{n}(x), \quad P_{0}=1, P_{1}=x-b_{0} .
$$

The Favard theorem [1] states that if $u_{n} \neq 0$, there exists a linear moment functional $\mathcal{L}$ such that

$$
\left\langle\mathcal{L}, P_{n}(x) P_{m}(x)\right\rangle=h_{n} \delta_{n m},
$$

where $h_{n}=u_{1} u_{2} \ldots u_{n}$ are normalization constants. The moment functional $\mathcal{L}$ is defined by its moments

$$
\left\langle\mathcal{L}, x^{n}\right\rangle=c_{n}, \quad n=0,1,2, \ldots
$$

In what follows we will assume that OP $P_{n}(x)$ are non-degenerate, i.e. $u_{n} \neq$ $0, n=1,2, \ldots$ This means, in particular, that $h_{n} \neq 0, n=0,1, \ldots$.

The Krall polynomials [10], are OP satisfying the eigenvalue problem

$$
L P_{n}(x)=\lambda_{n} P_{n}(x),
$$

where the $N$-th-order differential operator $L$ is defined by

$$
L=\sum_{k=0}^{N} a_{k}(x) \partial_{x}^{k},
$$

with $a_{k}(x)$ polynomials such that $\operatorname{deg}\left(a_{k}(x)\right) \leq k$. We use term "Krall polynomials" in a slightly more general sense than, say in [13]. Sometimes, the term "Bochner-Krall polynomials" is used instead [12].

It can be easily shown that $N$ must be even and $\lambda_{n}$ a polynomial in $n$ of degree $\leq N$.

The OP satisfying (1.4) for $N=2$ are the well known classical orthogonal polynomials [2]

Krall has found all possible OP satisfying 4-th order differential equations (i.e. $N=4)$. However for $N=6$ the problem of classifying of all Krall OP is so far unsolved (and only examples are known).

\footnotetext{
*Department of Mathematics and Statistics and Department of Physics, McGill University, 845 Sherbrooke St. W., Montreal, QC, Canada H3A 2T5 (vinet@vpa.mcgill.ca).

${ }^{\dagger}$ Department of Mathematics and Statistics, Concordia University, 7141 Sherbrooke W., Montreal, Que., Canada H4B 1R6 (yermolae@crm.umontreal.ca).

${ }^{\ddagger}$ Donetsk Institute for Physics and Technology, Donetsk, 83114, Ukraine (zhedanov@kinetic. ac.donetsk.ua).
} 
In all the explicit examples, one observes that the "non-classical" OP satisfying (1.4) differ from the classical ones (i.e. satisfying (1.4) with $N=2$ ) only by the presence of one or two concentrated masses at the endpoints of orthogonality interval. That is, all known Krall polynomials connected with the Jacobi polynomials belong to special classes of Koornwinder polynomials [9] having the following weight function on the interval $[0,1]$

$$
w(x)=x^{a}(1-x)^{b}+M_{0} \delta(x)+M_{1} \delta(1-x),
$$

Analogously, Krall's polynomials connected with the Laguerre polynomials have weight function $w(x)=x^{a} \exp (-x)+M \delta(x), \quad j=0,1,2, \ldots$ Koornwinder conjectured [9] that all OP associated to the weight function (1.6) should satisfy the differential equation (1.4) for some even $N$. However, it was shown in [21] that (when $M_{0} M_{1} \neq 0$ ) a necessary condition for this is that both $a$ and $b$ be simultaneously integer or half-integer numbers.

It was shawn in [6] that Koornwinder's polynomials with $a=b=j=0,1,2, \ldots$. and $M_{0}=M_{1}$ do satisfy differential equation (1.4) of order $N=2 j+4$.

We proposed in [21] a new method to generate Krall's polynomials. This method allowed one to construct a new family of Krall's polynomials having weight function $w(x)=x^{j}(1-x)^{b}+M \delta(x)$ with $b \geq-1 / 2$ an arbitrary parameter, $j=0,1,2, \ldots$ and $M$ an arbitrary mass.

In this paper we find another set of Krall polynomials that are a subclass of Koornwinder's polynomials. Namely, we show that the OP corresponding to the weight function (1.6) with $a=j=0,1,2, \ldots, b=0$ and with $M_{0}, M_{1}$ arbitrary, satisfy a differential equation (1.4) of order $N=2 j+6$. The simplest member of this family corresponding to $j=0$ and satisfying 6 -th order differential equation was found by Krall [13].

In the next section we briefly describe the method proposed in [21] and additional observations on the necessary and sufficient conditions for OP to satisfy equation (1.4).

Acknowlegement. The authors are grateful to V. Spiridonov for discussion and R. Koekoek and K. Kwon for sending their works before publication. The work of L. V. is supported in part through funds provided by NSERC (Canada) and FCAR (Quebec). A. Zh. thanks Centre de recherches mathématiques of the Université de Montréal for hospitality.

2. An algebraic approach to Krall polynomials. Without loss of generality we can put [21]

$$
a_{0}=0 .
$$

This means in particular that

$$
\lambda_{0}=0
$$

The polynomials $a_{n}(x)$ can be written in the form

$$
a_{n}(x)=\sum_{s=0}^{n} \alpha_{n s} x^{s}, \quad n=0,1, \ldots, N,
$$

where $\alpha_{n s}$ are some coefficients. Clearly $\operatorname{deg}\left(a_{n}(x)\right) \leq n$ and there exists at least one $m(0 \leq m \leq N)$ such that $\operatorname{deg}\left(a_{m}(x)\right)=m$. 
Consider the action of the operator $L$ on the monomials $x^{n}$ :

$$
L\left\{x^{n}\right\}=\sum_{k=0}^{n} A_{n k} x^{k}
$$

The coefficients $A_{n k}$ are the matrix elements of the operator $L$ in the monomial basis. These coefficients read

$$
A_{n k}=\sum_{j=0}^{N-n+k} \alpha_{j+n-k, j} n(n-1) \ldots(1+k-j) .
$$

It is useful to introduce new coefficients $A_{n}^{(s)}=A_{n, n-s}$. We have

$$
A_{n}^{(s)}=n(n-1)(n-2) \ldots(n-s+1) \pi_{N-s}(n),
$$

where

$$
\pi_{N-s}(n)=\alpha_{s 0}+\sum_{j=1}^{N-s} \alpha_{s+j, j}(n-s)(n-s-1) \ldots(n-s-j+1)
$$

is a polynomial in $n$ of degree not exceeding $N-s$.

The following statements are important in the theory of Krall polynomials [21].

Proposition 2.1. Assume that the differential operator $L$ have the representation coefficents $A_{n}^{(s)}$ with the only restriction that $A_{n}^{(0)} \neq A_{m}^{(0)}$ if $n \neq m$. There then exists a unique system of polynomials $P_{n}(x), n=0,1,2 \ldots$ satisfying equation (1.4) with

$$
\lambda_{n}=A_{n}^{(0)}
$$

Remark. In general, these polynomials $P_{n}(x)$ are not orthogonal.

Proposition 2.2. Assume that $A_{n}^{(s)}$ are arbitrary coefficients as in (2.6), with $N$ an even positive integer and $\pi_{N-s}(n)$ arbitrary polynomials of degree not exceeding $N-s$. At least one of these polynomials has degree $N-s$. Assume also that $A_{n}^{(s)}=$ $0, s>N$. There then exists a unique $N$-th order differential operator $L$ having $A_{n}^{(s)}$ as representation coefficients. (The order $N$ of this operator coincides with the maximal degree of all coefficients $A_{n}^{(s)}$ considered as polynomials in $\left.n\right)$.

The coefficients $\alpha_{i k}$ of the polynomials $a_{i}(x)$ in definition (1.5) of the differential operator $L$ are expressed as [21]

$$
\alpha_{s+k, k}=\left.\frac{\Delta^{k} \pi_{N-s}(n)}{k !}\right|_{n=s}
$$

where the difference operator $\Delta$ is defined as $\Delta F(n)=F(n+1)-F(n)$. Hence the differential operator $L$ can be reconstructed from these coefficients using (1.5) and (2.3).

Let $B_{n k}$ be the expansion coefficients of the polynomial $P_{n}(x)$

$$
P_{n}(x)=\sum_{k=0}^{n} B_{n k} x^{k}
$$


Substituting (2.10) and (2.4) into (1.4) we get

$$
\sum_{s=k}^{n} B_{n s} A_{s k}=\lambda_{n} B_{n k}, \quad k=n-N, n-N+1, \ldots, n .
$$

We thus have $N+1$ equations (2.11) for the coefficients $B_{n k}$ in terms of the known coefficients $A_{n k}$.

Proposition 2.3. The coefficients $B_{n k}$ are rational functions of the argument $n$.

The proof of this proposition can be found in [21] and will not be repeated here.

The relation (2.11) can be presented in the algebraic (matrix) form

$$
\mathbf{B A}=\Lambda \mathbf{B},
$$

where $\mathbf{A}$ and $\mathbf{B}$ are infinite-dimensional lower-triangular matrices with entries $A_{i k}$ and $B_{i k}$, whereas $\boldsymbol{\Lambda}$ is diagonal matrix with entries $\boldsymbol{\Lambda}_{i k}=\lambda_{i} \delta_{i k}$.

For our purposes the inverse problem is important: assume that some orthogonal polynomials $P_{n}(x)$ are given. The coefficients $B_{n k}$ are then known explicitly. We would like to find the matrix A. From (2.12) we have, formally:

$$
\mathbf{A}=\mathbf{B}^{-1} \Lambda \mathbf{B} \text {. }
$$

Note that the inverse matrix $\mathbf{B}^{-1}$ does exist. Indeed, we have the expansion

$$
x^{n}=\sum_{k=0}^{n} \mathbf{B}_{n k}^{-1} P_{k}(x)
$$

with unique coefficients $\mathbf{B}_{n k}^{-1}$. It is hence sufficient to choose the diagonal matrix $\boldsymbol{\Lambda}$, for the matrix $\mathbf{A}$ is then given by (2.13). Clearly, for arbitrary $\mathbf{B}$ and $\boldsymbol{\Lambda}$ one cannot expect that the elements $A_{n k}$ of the matrix $\mathbf{A}$ obtained in this way, have the required properties (2.6). As we will prove possible in some examples, one can hope that the coefficients $\lambda_{n}$ can be appropriately chosen so as to yield simple expressions for the coefficients $A_{n k}$. The algorithm for reconstructing $A_{n}^{(s)}$ works as follows [21]. It is convenient to rewrite (2.12) in the form

$$
\sum_{i=0}^{s} B_{n}^{(s-i)} A_{n-s+i}^{(i)}=\lambda_{n} B_{n}^{(s)}, \quad s=0,1,2, \ldots
$$

For $s=0$ we get the relation $A_{n}^{(0)}=\lambda_{n}$, which is not a restriction but rather a definition of $\lambda_{n}$.

For $s=1$ we have from $(2.15)$

$$
A_{n}^{(1)}=\left(\lambda_{n}-\lambda_{n-1}\right) B_{n}^{(1)}=\Omega_{n} B_{n}^{(1)},
$$

where

$$
\Omega_{n}=\lambda_{n}-\lambda_{n-1} .
$$

From (2.16) we have a restriction for $\lambda_{n}$. Indeed, both $\lambda_{n}$ and $A_{n}^{(1)}$ should be polynomials in $n$, while $B_{n}^{(1)}$ is a rational function:

$$
B_{n}^{(1)}=\frac{Q^{(1)}(n)}{R^{(1)}(n)}
$$


where $Q^{(1)}(n)$ and $R^{(1)}(n)$ are some polynomials in $n$ having no common zeroes.

It follows that necessarily

$$
\Omega_{n}=r(n) R^{(1)}(n)
$$

where $r(n)$ is a polynomial in $n$. Assume that $\Omega_{n}$ is already known and reads

$$
\Omega_{n}=\sum_{k=0}^{M} \omega_{k} n^{k}
$$

where $\omega_{k}$ are some coefficients. we can then obtain ann explicit expression for $\lambda_{n}$

$$
\lambda_{n}=\sum_{k=0}^{M} \omega_{k} J_{k}(n),
$$

where

$$
J_{k}(n)=\sum_{j=1}^{n} j^{k} .
$$

From (2.19) and (2.21), we get

$$
\operatorname{deg}\left(\lambda_{n}\right)=\operatorname{deg}(r(n))+\operatorname{deg}\left(R^{(1)}(n)\right)+1 \leq N,
$$

where $N$ is order of the differential operator $L$.

For $A_{n}^{(1)}$, we have from (2.16) and (2.18)

$$
A_{n}^{(1)}=r(n) Q^{(1)}(n) .
$$

For $s=2$ we get

$$
A_{n}^{(2)}=\left(\Omega_{n}+\Omega_{n-1}\right) B_{n}^{(2)}-\Omega_{n-1} B_{n}^{(1)} B_{n-1}^{(1)} .
$$

This process can be iterated: if $A_{n}^{(1)}, \ldots, A_{n}^{(s-1)}$ have already been found, $A_{n}^{(s)}$ is then uniquely determined from (2.15). However in order for the polynomials $P_{n}(x)$ to obey the eigenvalue equation (1.4), the coefficients $A_{n}^{(s)}$ should reads as in (2.6) or, equivalently,

$$
A_{n}^{(s)}=n(n-1)(n-2) \ldots(n-s+1) \pi_{N-s}(n), \quad s=0,1, \ldots, N,
$$

where $\pi_{k}(n)$ is a polynomial of degree not exceeding $k$. Moreover, we necessarily have

$$
A_{n}^{(s)} \equiv 0, \quad \text { for } \quad s>N
$$

The following criterion takes place [21].

Proposition 2.4. Assume that the coefficients $A_{n}^{(s)}$ are constructed via the algorithm (2.24), (2.25), ... The conditions (2.26) and (2.27) prove necessary and sufficient for the polynomials $P_{n}(z)$ to obey the eigenvalue equation (1.4).

There is, however, a more convenient criterion for OP to satisfy (1.4). This criterion will be obtained in the next section using technique of linear functionals. 
3. Criterion in terms of moments. Let $P_{n}(x)$ be monic polynomials that are solutions of (1.4) for some operator $L$. These polynomials need not be orthogonal. Consider a space of linear functionals $\sigma$ defined on the space of polynomials by their moments $\left\langle\sigma, x^{n}\right\rangle=c_{n}$.

The functionals $p(x) \sigma$ (where $p(x)$ is a polynomial) and $\partial_{x} \sigma$ are defined by [15]

$$
\begin{aligned}
& \langle p(x) \sigma, f(x)\rangle=\langle\sigma, p(x) f(x)\rangle \\
& \left\langle\partial_{x} \sigma, f(x)\right\rangle=-\left\langle\sigma, f^{\prime}(x)\right\rangle,
\end{aligned}
$$

where $f(x)$ is an arbitrary polynomial.

There exists a dual sequence of functionals $[15] \sigma_{n}, n=0,1,2, \ldots$ such that

$$
\left\langle\sigma_{n}, P_{m}(x)\right\rangle=\delta_{n m} .
$$

We can expand the functional $\sigma_{n}=\sum_{k=n}^{\infty} S_{k n} \rho_{k}$ in terms of the elementary functional basis $\rho_{n}$, where

$$
\left\langle\rho_{n}, x^{m}\right\rangle=\delta_{n m}
$$

From (3.3) and (2.10) we see that the moment matrix $\mathbf{S}$ is inverse of $\mathbf{B}$ :

$$
S_{i k}=\mathbf{B}_{i k}^{-1} .
$$

Define the conjugate operator $L^{*}$ (acting on the space of linear functionals $\sigma$ ) by the relation

$$
\left\langle L^{*} \sigma, f(x)\right\rangle=\langle\sigma, L f(x)\rangle,
$$

where $f(x)$ is any polynomial.

Proposition 3.1. If the polynomials $P_{n}(x)$ satisfy eigenvalue equation (1.4) then the dual functionals $\sigma_{n}$ satisfy the eigenvalue equations

$$
L^{*} \sigma_{n}=\lambda_{n} \sigma_{n}
$$

Proof. We have from (3.6), (3.3) and (1.4) that

$$
\left\langle L^{*} \sigma_{n}, P_{m}(x)\right\rangle=\left\langle\sigma_{n}, L P_{m}(x)\right\rangle=\lambda_{n} \delta_{n m} .
$$

But the relation (3.8) means that the set of functionals $L^{*} \sigma_{n}$ is dual with respect to the set $P_{n}(x)$, so we have (3.7).

Assume now that $P_{n}(x)$ are orthogonal polynomials. It is well known (see, e.g. [15]) that $P_{n}(x)$ are OP if and only if

$$
\sigma_{n}=h_{n}^{-1} P_{n}(x) \sigma_{0},
$$

where $h_{n}$ are some constants. In fact, these constants coincide with the normalization constants: $h_{n}=\left\langle\sigma_{0}, P_{n}^{2}(x)\right\rangle$. In this case the functional $\sigma_{0}$ plays the role of orthogonality functional for the OP $P_{n}(x)$ :

$$
\left\langle\sigma_{0}, P_{n}(x) P_{m}(x)\right\rangle=h_{n} \delta_{n m} .
$$

Let

$$
c_{n}=\left\langle\sigma_{0}, x^{n}\right\rangle
$$


be the moments corresponding to the orthogonality functional $\sigma_{0}$.

If the polynomials $P_{n}(x)$ are orthogonal, the relation (3.7) is rewritten as

$$
L^{*}\left\{P_{n}(x) \sigma_{0}\right\}=\lambda_{n} P_{n}(x) \sigma_{0}=\left(L P_{n}(x)\right) \sigma_{0}, \quad n=0,1,2, \ldots
$$

or, equivalently,

$$
L^{*}\left\{f(x) \sigma_{0}\right\}=L\{f(x)\} \sigma_{0} .
$$

The relation (3.13) holds for arbitrary polynomial $f(x)$. This relation can be rewritten in the equivalent form

$$
\left\langle\sigma_{0}, f(x) L\{\psi(x)\}\right\rangle=\left\langle\sigma_{0}, \psi(x) L\{f(x)\}\right\rangle .
$$

which holds for two arbitrary polynomials $f(x)$ and $\psi(x)$. Choosing $f(x)=x^{n}$, $\psi(x)=x^{m}$ and using (3.11) and (2.4) we rewrite (3.14) in the form

$$
I(n, m)=I(m, n)
$$

where $I(n, m)$ is the sum

$$
I(n, m)=\sum_{i=0}^{n} A_{n i} c_{m+i}
$$

The relation (3.15) should be valid for all values $m, n=0,1,2, \ldots$ In matrix form relation $(3.15)$ reads

$$
\mathbf{A C}=\mathbf{C A}^{T}
$$

where $\mathbf{A}^{T}$ is the transpose of $\mathbf{A}$, and $\mathbf{C}$ is the Hankel matrix with entries $\mathbf{C}_{i k}=c_{i+k}$.

Thus relation (3.15) is a necessary condition for the OP $P_{n}(x)$ to be Krall polynomials.

Conversely, let $P_{n}(x)$ be some OP with moments $c_{n}$. Assume that there exist coefficients $A_{n k}$ satisfying relation (3.15). Introduce a formal operator $L$ having $A_{n k}$ as its representative coefficients. (This operator need not be a differential operator). Then (3.15) is rewritten as

$$
\left\langle\sigma_{0}, x^{n} L\left\{x^{m}\right\}\right\rangle=\left\langle\sigma_{0}, x^{m} L\left\{x^{n}\right\}\right\rangle .
$$

Since $n, m$ are arbitrary, (3.18) is equivalent to (3.14). Choosing $f(x)=P_{n}(x)$, $\psi(x)=P_{m}(x)$ we have

$$
\left\langle\sigma_{0}, P_{n}(x) L P_{m}(x)\right\rangle=\left\langle\sigma_{0}, P_{m}(x) L P_{n}(x)\right\rangle .
$$

Obviously for any $n$ we have

$$
L P_{n}(x)=\sum_{i=0}^{n} \xi_{n i} P_{i}(x)
$$

with some coefficients $\xi_{n i}$. Substituting (3.20) into (3.19) we get

$$
\sum_{i=0}^{m} \xi_{m i}\left\langle\sigma_{0}, P_{n}(x) P_{i}(x)\right\rangle=\sum_{i=0}^{n} \xi_{n i}\left\langle\sigma_{0}, P_{m}(x) P_{i}(x)\right\rangle .
$$


Assume that $n>m$. Then the lhs of (3.21) vanishes, whereas the rhs is reduced to one term for $i=m$. From the orthogonality relation (3.10) we thus have

$$
\xi_{n m} h_{m}=0, \quad m=0,1, \ldots, n-1 .
$$

As $h_{m} \neq 0$, we have $\xi_{n m}=0, m=0,1, \ldots, n-1$ and hence

$$
L P_{n}(x)=\xi_{n n} P_{n}(x) \text {. }
$$

But condition (3.22) is equivalent to (1.4) with $\lambda_{n}=\xi_{n n}$.

We thus have the following result.

Proposition 3.2. Let $c_{n}$ be the moments corresponding to the OP $P_{n}(x)$. Assume that there exist coefficients $A_{n k}$ satisfying the relation (3.15). Then the polynomials $P_{n}(x)$ satisfy the eigenvalue equation (1.4), where $L$ is an abstract operator acting on monomials $x^{n}$ by (2.4). If, in addition, the coefficients $A_{n k}$ satisfy conditions (2.6) then $L$ is an $N$-th order differential operator.

Note that this proposition is valid for any operator $L$ having the property $(2.4)$ (e.g. $L$ can be q-difference operator [19]). We will call (3.15) the basic relation.

In what follows we will use the basic relation in order to verify that the coefficients $A_{n k}$ derived inductively from the first steps of the algorithm $(2.24),(2.25) \ldots$ do satisfy relation (3.15), and hence that the corresponding OP $P_{n}(x)$ satisfy the differential equation (1.4).

4. Koornwinder polynomials. The monic Jacobi polynomials are defined by the formula

$$
P_{n}^{(a, b)}(x)=\frac{(-1)^{n}(a+1)_{n}}{(a+b+n+1)_{n}} F_{1}\left(\begin{array}{c}
-n, n+a+b+1 \\
a+1
\end{array} ; x\right) .
$$

In what follows we will assume that $a \geq-1 / 2, b \geq-1 / 2$. The polynomials $P_{n}^{(a, b)}(x)$ are orthogonal on $(0,1)$

$$
\int_{0}^{1} P_{n}^{(a, b)}(x) P_{m}^{(a, b)}(x) w(x) d x=h_{n} \delta_{n m}
$$

with the normalized weight function

$$
w^{(a, b)}(x)=\frac{\Gamma(a+b+2)}{\Gamma(a+1) \Gamma(b+1)} x^{a}(1-x)^{b}
$$

(we define the Jacobi polynomials on the shifted orthogonality interval with respect to the standard one [8]). The moments of the Jacobi polynomials are

$$
c_{n}=\int_{0}^{1} w(x) x^{n} d x=\frac{(a+1)_{n}}{(a+b+2)_{n}} .
$$

We need also the values

$$
\begin{aligned}
P_{n}^{(a, b)}(0) & =\frac{(-1)^{n}(a+1)_{n}}{(a+b+n+1)_{n}} \\
Q_{n}^{(a, b)}(0) & =(-1)^{n+1} \frac{(a+b+1)(b+1)_{n} n !}{a(a+b+1)_{2 n}} \\
P_{n}^{(a, b)}(1) & =\frac{(b+1)_{n}}{(a+b+n+1)_{n}} \\
Q_{n}^{(a, b)}(1) & =\frac{(a+b+1)(a+1)_{n} n !}{b(a+b+1)_{2 n}}
\end{aligned}
$$


where $Q_{n}^{(a, b)}(z)$, the second-kind functions corresponding to the polynomials $P_{n}^{(a, b)}(x)$, are defined by the formula [18]

$$
Q_{n}^{(a, b)}(z)=\int_{0}^{1} \frac{P_{n}^{(a, b)}(x) w(x) d x}{z-x} .
$$

From (4.1) we find for the expansion coefficients $B_{n}^{(s)}$ :

$$
B_{n}^{(s)}=\frac{(-n)_{s}(-a-n)_{s}}{s !(-a-b-2 n)_{s}}
$$

Let us examine the polynomials

$$
\tilde{P}_{n}^{(a, b)}(x)=\mathcal{G}\left(0, \beta_{0}\right) \mathcal{G}\left(1, \beta_{1}\right) P_{n}^{(a, b)}(x)
$$

obtained from the Jacobi polynomials by the application of two Geronimus transforms $\mathcal{G}\left(0, \beta_{0}\right)$ and $\mathcal{G}\left(1, \beta_{1}\right)$ at the endpoints of orthogonality interval.

Recall [21] that the polynomials $\tilde{P}_{n}^{(a, b)}(x)$ have the expression

$$
\tilde{P}_{n}^{(a, b)}(x)=\kappa_{n}^{-1}\left|\begin{array}{ccc}
P_{n}(x) & P_{n-1}(x) & P_{n-2}(x) \\
\phi_{n}^{(0)} & \phi_{n-1}^{(0)} & \phi_{n-2}^{(0)} \\
\phi_{n}^{(1)} & \phi_{n-1}^{(1)} & \phi_{n-2}^{(1)}
\end{array}\right|,
$$

where $\kappa_{n}=\phi_{n-1}^{(0)} \phi_{n-2}^{(1)}-\phi_{n-1}^{(1)} \phi_{n-2}^{(0)}$ and where $\phi_{n}^{(0,1)}$ are defined by

$$
\begin{aligned}
& \phi_{n}^{(0)}=Q_{n}^{(a, b)}(0)+\beta_{0} P_{n}^{(a, b)}(0) \\
& \phi_{n}^{(1)}=Q_{n}^{(a, b)}(1)+\beta_{1} P_{n}^{(a, b)}(1)
\end{aligned}
$$

with $\beta_{0}, \beta_{1}$ arbitrary parameters.

The polynomials $\tilde{P}_{n}^{(a, b)}(x)$ are monic orthogonal polynomials with normalized weight function [21]

$$
\tilde{w}(x)=\mu\left(\frac{(a+b+1)(a+b)}{a b} w^{(a-1, b-1)}(x)-\beta_{0} \delta(x)+\beta_{1} \delta(x-1)\right),
$$

where $\mu^{-1}=(a+b)(a+b+1) /(a b)+\beta_{1}-\beta_{0}$ and $w^{(a, b)}(x)$ is given in (4.3).

Comparing (4.12) with (1.6), we arrive at the important relation

$$
\mathcal{G}\left(0, \beta_{0}\right) \mathcal{G}\left(1, \beta_{1}\right) P_{n}^{(a, b)}(x)=P_{n}^{\left(a-1, b-1 ; M_{0}, M_{1}\right)}(x),
$$

where

$$
\begin{aligned}
& M_{0}=-\beta_{0} \frac{a b}{(a+b)(a+b+1)} \\
& M_{1}=\beta_{1} \frac{a b}{(a+b)(a+b+1)} .
\end{aligned}
$$

Thus Koornwinder polynomials $P_{n}^{\left(a-1, b-1 ; M_{0}, M_{1}\right)}(x)$ can be viewed as doubly Geronimus transformed Jacobi polynomials.

The moments of the polynomials $\mathcal{G}\left(0, \beta_{0}\right) \mathcal{G}\left(1, \beta_{1}\right) P_{n}^{(a, b)}(x)$ are

$$
c_{n}=\mu\left(\frac{(a+b)(a+b+1)}{a b} \frac{(a)_{n}}{(a+b)_{n}}-\beta_{0} \delta_{n 0}+\beta_{1}\right), \quad n=0,1,2, \ldots
$$


As was shown in [21], the necessary condition for the polynomials $\mathcal{G}\left(0, \beta_{0}\right) \mathcal{G}\left(1, \beta_{1}\right) P_{n}^{(a, b)}(x)$ to satisfy the eigenvalue equation (1.4) is that both $a, b$ are simultaneously integers or half-integers.

In what follows we restrict ouselves to the case $a=1,2,3, \ldots, b=1$. Assume that the coefficients $A_{n}^{(s)}$ corresponding to the polynomials $\mathcal{G}\left(0, \beta_{0}\right) \mathcal{G}\left(1, \beta_{1}\right) P_{n}^{(a, 1)}(x)$ do exist. Then the expression (3.16) (where $c_{n}$ have the expression (4.14)) takes the form

$$
I(n, m)=\mu\left((a+1)(a+2) \sum_{s=0}^{n} \frac{A_{n}^{(s)}}{a+n+m-s}+\beta_{1} \sum_{s=0}^{n} A_{n}^{(s)}-\beta_{0} A_{n}^{(n)} \delta_{0 m}\right) .
$$

Note that the expansion coefficients $\tilde{B}_{n}^{(s)}$ for the polynomials $\mathcal{G}\left(0, \beta_{0}\right) \mathcal{G}\left(1, \beta_{1}\right) P_{n}^{(a, b)}(x)$ are easily found from (4.10) and (4.11):

$$
\begin{aligned}
& \tilde{B}_{n}^{(0)}=1 \\
& \tilde{B}_{n}^{(1)}=B_{n}^{(1)}-D_{n}^{(1)} \\
& \tilde{B}_{n}^{(s)}=B_{n}^{(s)}-D_{n}^{(1)} B_{n-1}^{(s-1)}+D_{n}^{(2)} B_{n-2}^{(s-2)}, \quad s=2,3, \ldots, n,
\end{aligned}
$$

where

$$
\begin{aligned}
& D_{n}^{(1)}=\frac{\phi_{n}^{(0)} \phi_{n-2}^{(1)}-\phi_{n}^{(1)} \phi_{n-2}^{(0)}}{\phi_{n-1}^{(0)} \phi_{n-2}^{(1)}-\phi_{n-1}^{(1)} \phi_{n-2}^{(0)}}, \\
& D_{n}^{(2)}=\frac{\phi_{n}^{(0)} \phi_{n-1}^{(1)}-\phi_{n}^{(1)} \phi_{n-1}^{(0)}}{\phi_{n-1}^{(0)} \phi_{n-2}^{(1)}-\phi_{n-1}^{(1)} \phi_{n-2}^{(0)}} .
\end{aligned}
$$

5. Reconstruction of the coefficients $A_{n}^{(s)}$. In this section we show that the coefficients $A_{n}^{(s)}$ satsfying relation (3.15) do exist for the polynomials $\mathcal{G}\left(0, \beta_{0}\right) \mathcal{G}\left(1, \beta_{1}\right) P_{n}^{(a, 1)}(x)$, where $a=1,2, \ldots$ In order to reconstruct these coefficients we start from relation $(2.24)$ and choose $r(n)=1$, we then reconstruct $A_{n}^{(2)}$ using (2.25) etc. Repeating this process we can guess that $A_{n}^{(s)}$ has the expression

$$
A_{n}^{(s)}=W_{n}^{(s)}+\beta_{1} X_{n}^{(s)}+\beta_{0} Y_{n}^{(s)}+\beta_{0} \beta_{1} Z_{n}^{(s)}
$$

where

$$
\begin{aligned}
& W_{n}^{(s)}=-(-1)_{s}(a+2) !^{2} \frac{n(n+a-s)}{a} \\
& X_{n}^{(s)}=-(-2)_{s}(a-1) !(a+2) ! \times \\
& \frac{(-n)_{s}(n+1)_{1-s}(n+a-s)\left(n^{2}+(a-1) n+2 s-s^{2}\right)}{2 s !} \\
& Y_{n}^{(s)}=\frac{(a+2)(-1-a)_{s}(1-n)_{s}(n)_{a+1}^{2}}{s !(-a-n)_{s}} \\
& Z_{n}^{(s)}=(-s(s-1)+(a+1)(n-1)(n+a+1)+(a+1)(2-n) s) \times \\
& \frac{(n)_{a+1}^{2}(-a-2)_{s}(1-n)_{s}}{(a+1)(a+2) s !(-a-n)_{s}}
\end{aligned}
$$

In the next section we show that the condition (3.15) is satisfied and hence that the coefficients $A_{n}^{(s)}$ given by $(5.1)$ do correspond to a differential operator having the polynomials $\mathcal{G}\left(0, \beta_{0}\right) \mathcal{G}\left(1, \beta_{1}\right) P_{n}^{(a, 1)}(x)$ as eigenfunctions. 
6. Verification of the basic relation. We should verify that $I(n, m)=I(m, n)$ with $I(n, m)$ given by $(4.15)$. Note that when $m=0$ the basic relation reduces to

$$
(a+1)(a+2) \sum_{s=0}^{n} \frac{A_{n}^{(s)}}{a+n-s}+\beta_{1} \sum_{s=0}^{n} A_{n}^{(s)}-\beta_{0} A_{n}^{(n)}=0, \quad n=0,1,2, \ldots
$$

Introduce the sums

$$
S_{1}(n)=\sum_{s=0}^{n} A_{n}^{(s)}
$$

and

$$
S_{2}(n, m)=\sum_{s=0}^{n} \frac{A_{n}^{(s)}}{a+n+m-s}
$$

Then the basic relation reads

$$
\begin{gathered}
(a+1)(a+2) S_{2}(n, m)+\beta_{1} S_{1}(n)=(a+1)(a+2) S_{2}(m, n)+\beta_{1} S_{1}(m) \\
m>0, n=0,1,2,3, \ldots
\end{gathered}
$$

The sum $S_{1}(n)$ can be calculated using the Chu-Vandermonde identity [2]

$$
{ }_{2} F_{1}\left(\begin{array}{c}
-n, \alpha \\
\beta
\end{array} ;\right)=\frac{(\beta-\alpha)_{n}}{(\beta)_{n}}, n=0,1,2, \ldots
$$

The sum $S_{2}(n, m)$ for $m>0$ can be calculated using the formula

$$
{ }_{3} F_{2}\left(\begin{array}{c}
-n, \alpha, \beta \\
\alpha+1, \beta-k
\end{array} ; 1\right)=\left\{\begin{array}{c}
\frac{n !(1+\alpha-\beta)_{k}}{(\alpha+1)_{n}(1-\beta)_{k}}, k=0,1, \ldots, n \\
\frac{\alpha n !}{(1-\beta)_{n+1}}\left(\frac{(1+\alpha-\beta)_{n+1}}{(\alpha)_{n+1}}-1\right), k=n+1
\end{array}\right.
$$

Formula (6.7) can be easily derived using well known transformation formulas for the hypergeometric function ${ }_{3} F_{2}(1)[2]$ : in our case, the lhs of $(6.7)$ can be reduced to ${ }_{2} F_{1}(1)$, which can be calculated from (6.6). For $m=0$ the sum $S_{2}(n, 0)$ is calculated using (6.6).

We thus have for $S_{1}(n)$

$$
S_{1}(n)=\beta_{0}(a+2) !(n)_{a+1}-n(a+2) !^{2} / a
$$

The sum $S_{2}(n, m)$ has a more complicated expression. For $m>0$ we have

$$
\begin{gathered}
S_{2}(n, m)=\beta_{0} \beta_{1} a !(n)_{a+1}\left(\frac{(m)_{a+1}\left(n^{2}+m^{2}+(3+a) m n-3(m+n)+1-a\right)}{(n+m-2)_{a+3}}-1\right) \\
+\beta_{1} \frac{(a-1) !(a+2) ! n(n+a-1)\left(n^{2}-m-2 a-2 n+2 a n+2 m n+a^{2}+a m\right)}{(a+n+m)(a+m+n-1)(a+n+m-2)} \\
+\beta_{0} \frac{(a+2) !(n)_{a+1}(m)_{a+1}}{(n+m-1)_{a+2}}-\frac{n m(a+2) !^{2}}{a(n+m+a)(n+m+a-1)}
\end{gathered}
$$


For $m=0$ one has

$$
\begin{aligned}
& S_{2}(n, 0)=\beta_{0} \beta_{1} a !\left(-(n)_{a+1}+(a+1) !\left(\delta_{n 1}-\delta_{n 2}\right)\right) \\
&+ \beta_{1} n(a-1) !(a+2) !+\beta_{0} a !(a+2) ! \delta_{n 1}
\end{aligned}
$$

Finally we need the expression for $A_{n}^{(n)}$ :

$$
A_{n}^{(n)}=(a+2) !\left((a+2) ! \delta_{n 1}+\beta_{1}(a+1) !(a+2) !\left(\delta_{n 1}-\delta_{n 2}\right)\right) .
$$

Combining expressions (6.8), (6.9), (6.10) and (6.11) we see that basic relations (6.4), (6.5) are identically satisfied.

Moreover, the coefficients $A_{n}^{(s)}$ satisfy the properties of the proposition $\mathbf{2 . 2}$ with $N=2 a+4$. We thus have

Proposition 6.1. The polynomials $\mathcal{G}\left(0, \beta_{0}\right) \mathcal{G}\left(1, \beta_{1}\right) P_{n}^{(a, 1)}(x)$ with $a=1,2,3 \ldots$ and $\beta_{0}, \beta_{1}$ arbitrary satisfy the eigenvalue equation (1.4) where $L$ is a differential operator of order $2 a+4$.

Using relation (4.13) we arrive at the

Proposition 6.2. Koornwinder's polynomials $P_{n}^{\left(a, 0 ; M_{0}, M_{1}\right)}(x)$ with $a=0,1,2$, $\ldots$ and arbitrary masses $M_{0}, M_{1}$ inserted at the endpoints $x=0,1$, satisfy the differential equation (1.4) of order $2 a+6$.

Note that for $a=0$ we return to the example of Krall polynomials satisfying a 6 -th order differential equation [13], [14]. For $a>0$ our result is perhaps new.

The coefficients $\alpha_{i k}$ of the differential operator $L$ can be reconstructed via (2.9). The expression for the polynomials $a_{k}(x)$ in (1.5) is rather complicated. However for $k=N=2 a+4$ and $k=N-1=2 a+3$ we have the simple expressions

$$
\begin{aligned}
& a_{N}(x)=\frac{\beta_{0} \beta_{1}}{a+2} x^{a+2}(x-1)^{a+2} \\
& a_{N-1}(x)=\beta_{0} \beta_{1} x^{a+1}(x-1)^{a+1}(3(a+1) x-2 a-1) .
\end{aligned}
$$

Note added in proof.

Results related to those presented here can now be found in a paper [7] that has appeared after the submission of this article.

It is shown in [7] that for positive weight functions of the type (1.6), a finite-order differential equation exists if and only if

(i) both masses $M_{0}, M_{1}$ are arbitrary positive and parameters $a, b$ are nonnegative integers. In this case the order of the differential equation is $2 a+2 b+6$;

(ii) $M_{1}=0$ and $M_{0}$ is arbitrary positive. Parameter $b>-1$ is arbitrary and $a=0,1, \ldots$. In this case the order of the differntial equation is $2 a+4$.

(iii) $M_{0}=0$ and $M_{1}$ is arbitrary positive. Parameter $a>-1$ is arbitrary and $b=0,1, \ldots$ In this case the order of the differntial equation is $2 b+4$.

In all the above cases the differential equations were presented explicitly in [7].

We considered the case (ii) in [21] and in the present paper we consider a special case of (i) when $b=0$ and $a=0,1,2, \ldots$ The interest of our results lie in particular in the fact that we use a different method to construct the explicit operators. This method was proposed in [21] and was recently applied to investigate q-Krall polynomials [19]. 


\section{REFERENCES}

[1] T. Chinara, An Introduction to Orthogonal Polynomials, Gordon and Breach, NY, 1978.

[2] A. ERdÉlyi et Al. (Eds.), Higher Transcendental Functions, Bateman Manuscript Project, Vol. I, McGraw-Hill, New York, 1953.

[3] YA. L. Geronimus, On the polynomials orthogonal with respect to a given number sequence, Zap. Mat. Otdel. Khar'kov. Univers. i NII Mat. i Mehan., 17 (1940), pp. 3-18.

[4] Ya. L. Geronimus, On the polynomials orthogonal with respect to a given number sequence and a theorem by W. Hahn, Izv. Akad. Nauk SSSR, 4 (1940), pp. 215-228.

[5] J. Koекоек And R. Koekoek, On a differential equation for Koornwinder's generalized Laguerre polynomials, Proc. Amer. Math. Soc., 112 (1991), pp. 1045-1054.

[6] R. Koековк, Differential equations for symmetric generalized ultraspherical polynomials, Trans. Amer. Math. Soc., 345 (1994), pp. 47-71.

[7] J. Koекоек And R. Kozkozk, Differential equations for generalized Jacobi polynomials, J. Comput. and Appl. Math., 126 (2000), pp. 1-31.

[8] R. Koekoek And R. F. Swarttouw, The Askey scheme of hypergeometric orthogonal polynomials and its q-analogue, Report 94-05, Faculty of Technical Mathematics and Informatics, Delft University of technology, 1994.

[9] T. H. KoORNWINDER, Orthogonal polynomials with weight function $(1-x)^{\alpha}(1+x)^{\beta}+M \delta(x+$ 1) $+N \delta(x-1)$, Can. Math. Bull., 27 (1984), pp. 205-214.

[10] H. L. Krall, Certain differential equations for Tchebychev polynomials, Duke Math. J., 4 (1938), pp. 705-719.

[11] K. H. Kwon, L. L. Littlejohn, And B. H. Yoo, Characterization of orthogonal polynomials satisfying differential equations, SIAM J. Math. Anal., 25 (1994), pp. 976-990.

[12] K. H. Kwon, G. H. Yoon, And L. L. Littlejohn, Bochner-Krall polynomials, preprint.

[13] L. L. Littlejohn, The Krall polynomials: A new class of orthogonal polynomials, Quaestiones Math., 5 (1982), pp. 255-265.

[14] L. L. Littlejohn and A. M. Krall, Orthogonal polynomials and higher order singular SturmLiouville systems, Acta Applicande Math., 17 (1989), pp. 99-170.

[15] P. Maroni, Variations around classical orthogonal polynomials. Connected problems, J. Comput. and Appl. Math., 48 (1993), pp. 133-155.

[16] V. Spiridonov And A. Zhedanov, Discrete Darboux transformation, discrete-time Toda lattice and the Askey-Wilson polynomials, Methods and Appl. of Analysis, 2 (1995), pp. 369-398.

[17] V. Spiridonov, L. Vinet, AND A. Zhedanov, Spectral transformations, self-similar reductions and orthogonal polynomials, J. Phys. A: Math. \& Gen., 30 (1997), pp. 7621-7637.

[18] G. Szegö, Orthogonal Polynomials, fourth edition, AMS, 1975.

[19] L. Vinet AND A. Zhedanov, Generalized little q-Jacobi polynomials as eigensolutions of higherorder q-difference operators, Proc. Amer. Math. Soc., 129 (2001), pp. 1317-1327.

[20] A. Zhedanov, Rational spectral transformations and orthogonal polynomials, J. Comput. and Appl. Math., 85 (1997), pp. 67-86.

[21] A. Zhedanov, A method of constructing Krall's polynomials, J. Comput. and Appl. Math., 107 (1999), pp. 1-20. 
